\title{
Proposed Next Generation GRB Mission: EXIST
}

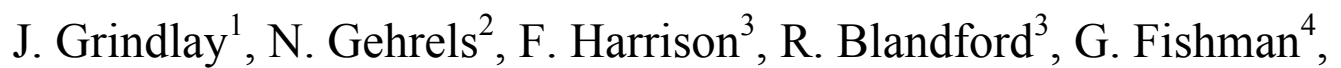 \\ C. Kouveliotou ${ }^{4}$ D.H. Hartmann ${ }^{5}$, S. Woosley ${ }^{6}$, W. Craig ${ }^{7}$ and J. Hong ${ }^{1}$ \\ 1. Harvard, 2. NASA/GSFC, 3. Caltech, 4. NASA/MSFC, 5. Clemson Univ., 6. UC Santa Cruz, 7. LLNL
}

\begin{abstract}
A next generation Gamma Ray Burst (GRB) mission to follow the upcoming Swift mission is described. The proposed Energetic X-ray Imaging Survey Telescope, EXIST, would yield the limiting (practical) GRB trigger sensitivity, broad-band spectral and temporal response, and spatial resolution over a wide field. It would provide high resolution spectra and locations for GRBs detected at $\mathrm{GeV}$ energies with GLAST. Together with the next generation missions Constellation-X, NGST and LISA and optical-survey (LSST) telescopes, EXIST would enable GRBs to be used as probes of the early universe and the first generation of stars. EXIST alone would give $\sim 10-50$ " positions (long or short GRBs), approximate redshifts from lags, and constrain physics of jets, orphan afterglows, neutrinos and SGRs.
\end{abstract}

\section{INTRODUCTION}

Gamma-ray bursts (GRBs) are the most luminous events since the Big Bang. Current models for at least the "long" GRBs favor their origin in the collapse of massive stars. As such, their study with the most powerful telescopes can map the universe back to the very first stars, believed to be very massive. GRBs from such large redshifts will require a next generation telescope to follow the Swift mission, currently planned for launch in 2003. The proposed Energetic $\mathrm{X}$-ray Imaging Survey Telescope, EXIST, can achieve these objectives as the Next Generation GRB mission.

We describe the current mission concept for EXIST and then some of the GRB and associated science that could be conducted with a nominal $5 y$ mission.

\section{EXIST MISSION CONCEPT}

The primary mission requirements for a next generation GRB mission are: 1. very large area, for maximum GRB sensitivity; 2. large field of view (FOV), for collection of a large GRB sample and sensitivity to rare events, including low-luminosity nearby GRBs possibly observed off-axis (e.g. SN1998bw); 3. high angular resolution and fine positional determination (10") for (near-) real time optical identifications; and 4. broad energy band coverage, with imaging response down to $\sim 10 \mathrm{keV}$ for high z GRBs and up to $\sim 600 \mathrm{keV}$ to extend beyond the $\sim 300 \mathrm{keV} v \mathrm{~F} v$ energy peak and maximize sensitivity to broad energy-dependent lags (hard-soft) which may allow measures of GRB luminosity-distance.

Broad-band ( 10-600 keV) hard x-ray imaging over a wide FOV is best conducted with a coded aperture telescope with an imaging detector capable of fine position resolution (to record the coded mask shadow), high $Z$ stopping power for good high energy sensitivity in moderate detector thickness, and compact mounting and tiling capability for extension to a very large area total detector array. Coded aperture imagers are background limited and so record signal to noise $\mathrm{S} / \mathrm{N} \sim\left(\mathrm{A}_{\operatorname{det}}{ }^{\circ} \mathrm{T} / \mathrm{B}\right)^{0.5}$, for a given detector area $A_{\text {det }}$ recording background $\mathrm{B}$ (cts $\mathrm{cm}^{-2} \mathrm{sec}^{-1}$ ) for a source observed for time $\mathrm{T}(\mathrm{sec})$. Optimum imaging sensitivity requires systematic variations on the detector (e.g., due to gain and non-uniform background variations) to be effectively averaged, which can best be achieved by continuously scanning the detector-telescope across the sky. Since for the wide-field $\left(>>10^{\circ}\right)$ imaging needed for GRB sample statistics, the background is dominated by the diffuse cosmic flux (primarily over $\sim 20-200 \mathrm{keV}$ ) recorded in the FOV of size $\theta \times \theta$, then $B \sim \theta^{2}$. Similarly, the exposed $\mathrm{A}_{\mathrm{det}}$, available integration time $\mathrm{T}$, and recorded $\mathrm{B}$ are each proportional to the angular width $\theta$ of the FOV in the scan direction. Thus, for a scan at orbital rate $\mathrm{d} \varphi / \mathrm{dt} \sim 4^{\mathrm{o}} \mathrm{min}^{-1}$, the expected $\mathrm{S} / \mathrm{N} \sim \theta^{0.5}$ for GRBs with duration $\mathrm{T}_{\mathrm{b}} \sim \theta(\mathrm{d} \varphi / \mathrm{dt})^{-1} \sim 3-10 \mathrm{~min}$ (e.g. long GRBs, or possibly high-z GRBs) and S/N independent of $\theta$ for typical GRBs $\left(\mathrm{T}_{\mathrm{b}}<1 \mathrm{~min}\right)$. Since

CP662, Gamma-Ray Burst and Afterglow Astronomy 2001: A Workshop Celebrating the First Year of the HETE Mission,

edited by G. R. Ricker and R. K. Vanderspek

(C) 2003 American Institute of Physics 0-7354-0122-5/03/\$20.00 
the total GRB sample $\mathrm{N}_{\mathrm{b}} \sim \theta^{2}$, large $\theta$ is optimum; this also maximizes the persistent source sensitivity.

These general considerations, as well as the primary goal to conduct a hard x-ray imaging survey which extends ROSAT sensitivity to $>100 \mathrm{keV}$ but with all sky coverage each orbit [1], yield the preliminary EXIST mission concept outlined below. The baseline implementation is for a Free Flyer mission although a version studied originally [1,2] could be mounted on the International Space Station.

\section{Mission Implementation Overview}

Three telescopes, each with a $60^{\circ} \times 75^{\circ}$ fully-coded FOV, are mounted on a base spacecraft as shown in Figure $1 \mathrm{a}$ to form a combined $180^{\circ} \times 75^{\circ}$ fan beam which images the full sky each orbit. Each telescope is constructed (Figure 1b) of a $3 \times 3$ array of actively collimated (CsI) overlapping FOV $\left(60^{\circ}\right.$ x 50 $)$ subtelescope modules, each read out with arrays of $\mathrm{Cd}$ $\mathrm{Zn}$-Te (CZT) detectors (5mm thick; $1.3 \mathrm{~mm}$ pixels) which view the sky through a curved coded aperture.

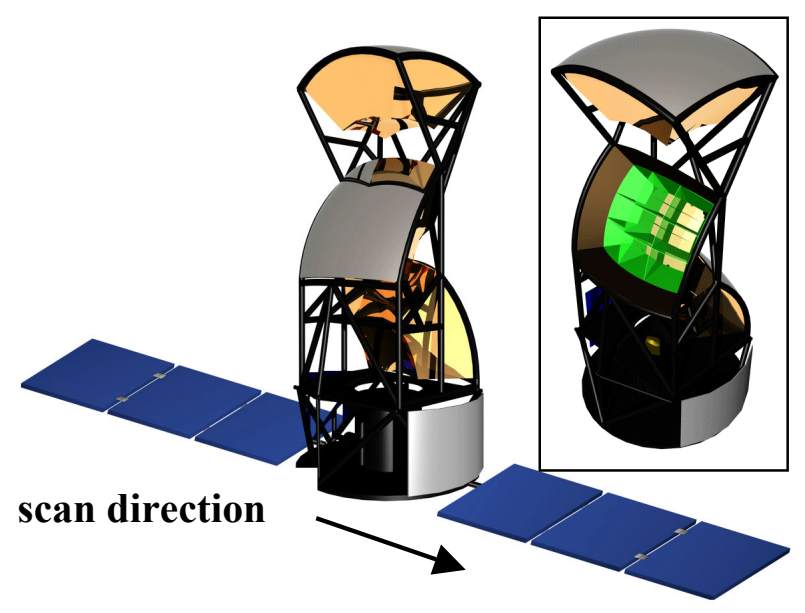

FIGURE 1. a) EXIST telescopes (3) on spacecraft, zenith pointed along orbital scan direction, b) cutaway view showing imaging CZT detector arrays and active collimator.

Parameters for the mission are summarized in Table 1 and estimated continuum and line sensitivities $(5 \sigma, \leq 1 \mathrm{y}$, depending on source orbital latitude) are shown in Figure 2. After the first year all-sky survey, or at any time for Targets of Opportunity, the mission would be operated as an Observatory, with an active guest observer program for pointed observations with the central telescope while the outer two telescopes continue the Survey. GRBs are imaged in the wide
Table 1: EXIST Mission Parameters

\begin{tabular}{|c|c|}
\hline Energy range & $10-600 \mathrm{keV}$ \\
\hline \multirow[t]{2}{*}{ FOV } & $180^{\circ} \times 75^{\circ}$ (fully coded) \\
\hline & $\sim 5$ steradians (partial coded) \\
\hline Angular Resolution & $2-5^{\prime}$ (10-50"source locations) \\
\hline Energy/Temporal Resolution & $1-3 \% ; 2 \mu \mathrm{sec}$ \\
\hline \multirow[t]{2}{*}{ Sensitivity $(5 \sigma, \leq 1 \mathrm{y})$} & $\sim 0.05 \mathrm{mCrab}(10-100 \mathrm{keV})$ \\
\hline & $\sim 0.5 \mathrm{mCrab}(>200 \mathrm{keV})$ \\
\hline Telescopes, Detectors & Coded aperture, $8 \mathrm{~m}^{2} \mathrm{CZT}$ \\
\hline Pointing, Aspect & $\sim 1^{\circ}$ stability, 5"knowledge \\
\hline Mass, Power, TM & $8500 \mathrm{~kg}, 1500 \mathrm{~W}, 1.5 \mathrm{Mbs}$ \\
\hline Launch, Cost (incl. Ops) & Delta IV, \$330M (w/ cont.) \\
\hline
\end{tabular}

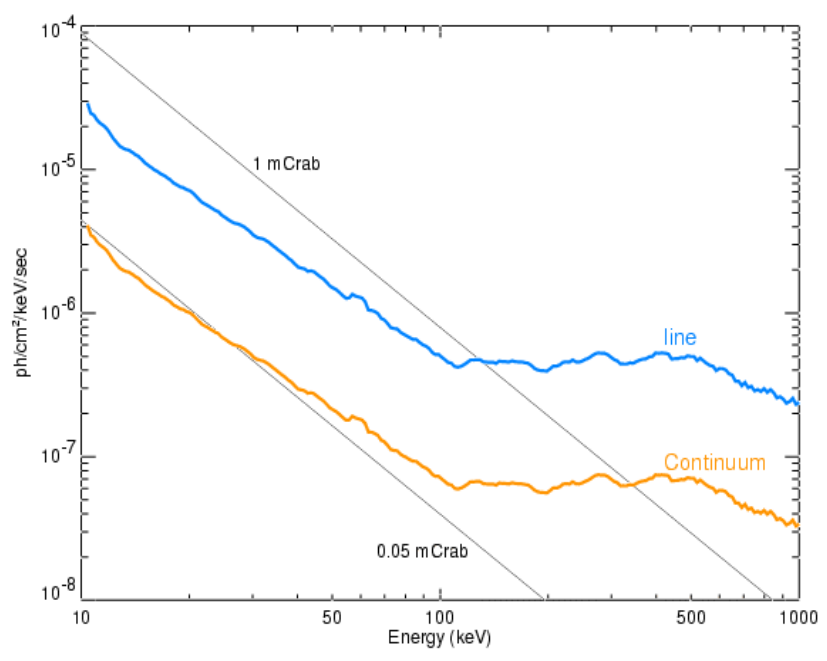

FIGURE 2. EXIST survey sensitivities. GRB sensitivities are $\sim 50 \mathrm{mCrab}$ for an assumed $10 \mathrm{sec}$ duration burst.

combined field $(5 \mathrm{sr})$ regardless of Survey or Observatory mode. The active collimator and rear shield ( $1 \mathrm{~cm}$ and $2 \mathrm{~cm}$ thick CsI, respectively) will be pulse-height-analyzed to extend GRB spectral coverage up to $\sim 1-3 \mathrm{MeV}$. Burst positions are derived to $\sim 1-3^{\prime}$ within 10 sec for rapid transmission to ground and other observatories, and to within $\sim 10-50 "$ in ground analysis of the full data within $\sim 3$ hours.

Primary Survey science is the study of obscured AGN and black holes on all scales. Details of the full mission and science will be given in a later paper. 


\section{GRB SCIENCE FROM EXIST}

EXIST is a Next Generation Burst Observatory. With sensitivity to weak events a factor $\sim 20$ below BATSE and $\sim 3-10$ below Swift (given the extended low and high energy band of EXIST), it should provide $\sim 10-50$ " locations for $2-3$ GRBs a day. With its large instantaneous field of view it can study both low luminosity nearby GRB events (like SN1998bw) as well as the brightest events most likely to be observed by gravitational wave and neutrino detectors. EXIST will be on orbit at an amazing time, when NGST will be studying the high-redshift universe and Contellation- $X$ will enable high resolution spectra of x-ray afterglows pinpointed by EXIST to be measured. LISA and LIGO2 will be providing the first sensitive gravitational wave detections, and IceCube, Auger, and other high-energy neutrino and ultra-high energy cosmic ray detectors will be operating. The coincidence of these capabilities with EXIST will provide opportunities to search for the first massive stars in the universe to very high redshifts and open up non-electromagnetic channels of GRB energy release for observation - challenging our theories of relativistic shocks and testing physics from special relativity to neutrino masses and couplings.

\section{First Massive Stars to EXIST}

Nearly 2/3 of all GRBs, the "long bursts", explode at significant cosmological distances. With its high sensitivity, EXIST can detect GRBs at high redshift ( $\sim$ 10-20; cf. Figure 3), enabling the first direct search

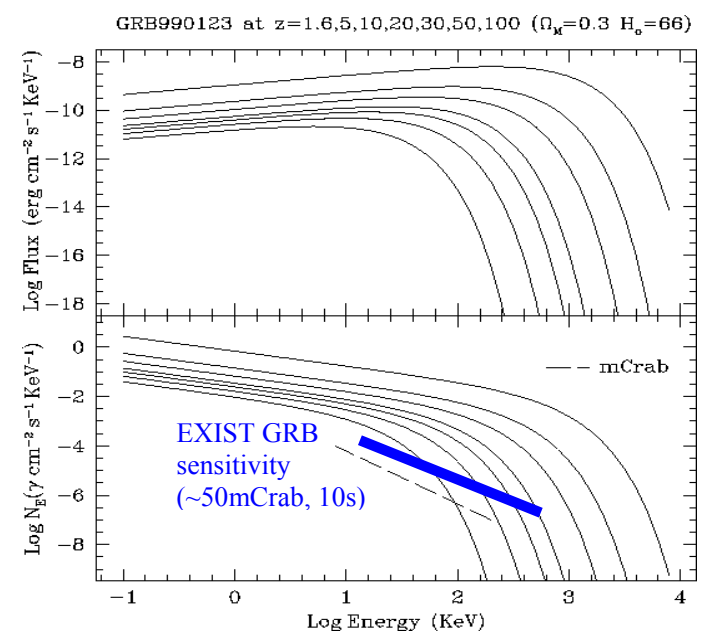

FIGURE 3. GRB sensitivity vs. z (adapted from [3]).

for the initial generation of stars (Pop III) that were likely very massive. Their resulting epoch of black hole $(\mathrm{BH})$ formation would, given increasing evidence for long burst $(>2 \mathrm{sec})$ GRB production by hypernovae in Collapsars [4, 5], produce an epoch of GRBs. Spectroscopy of their optical afterglows, detectable in the IR with NGST, would enable mapping cosmic structure back to the "dark ages" [6]. This requires a rapid estimate of GRB redshift, which could be provided by the "photometric redshifts" to be derived from the observed relation [7] (cf. Figure 4) between GRB hard-soft lags and absolute luminosity.

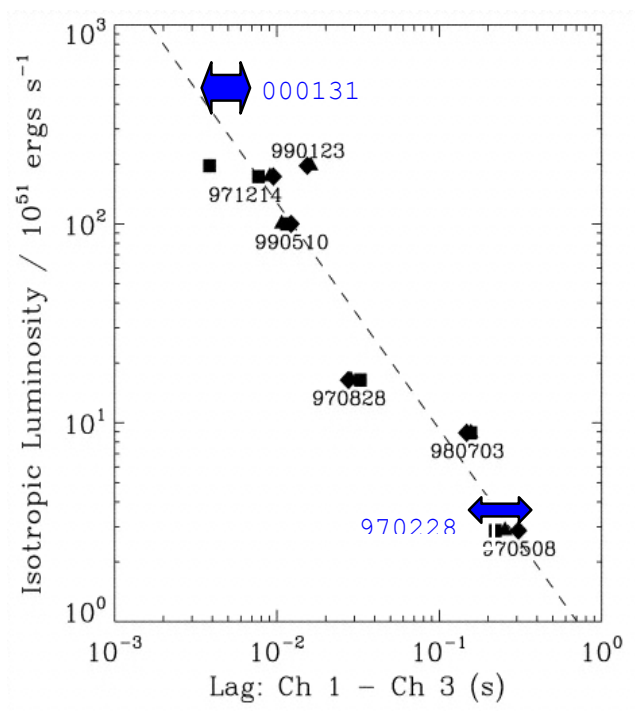

FIGURE 4. Luminosity-lag relation for GRBs (adapted from [7]). The observed correlation, $\mathrm{L}_{\mathrm{pk}} \propto \Delta \tau^{-1.14}$, may be understood as a kinematic effect from beaming [8].

Application of this technique to GRBs at high $\mathrm{z}$ requires the very large collection area for optimum statistics as well as the broad energy band coverage and good spectral resolution of EXIST.

The large GRB sample with approximate redshifts collected by EXIST will constrain the star formation rate to large z, complementing NGST. The EXIST deep sample will also provide possible GRB locations and redshifts for "orphan afterglows", probably found with SDSS [9] and expected in quantity from LSST in the EXIST era. Since orphans may preferentially be offaxis GRBs [10], they are likely to be x-ray bright and thus possibly related to the $\mathrm{x}$-ray flashes (XRFs) which appear to form a spectral extension to GRBs [11]. Alternatively, the XRFs may be GRBs from massive $\left(\sim 300 \mathrm{M}_{\odot}\right)$ Pop III stars at $\mathrm{z} \sim 10$ undergoing collapse to BHs, which also would produce background high energy $\left(\sim 10^{5} \mathrm{GeV}\right)$ neutrinos detectable with IceCube [12]. 


\section{Highest Energy Particles to EXIST}

The relativistic shocks driven into the interstellar medium after a GRB explosion can produce highenergy neutrinos in several components with energies $\sim 10 \mathrm{GeV}, \sim 100 \mathrm{TeV}$ and $\sim 10^{18} \mathrm{eV}$ on timescales of $<10$ s. Theory [13] predicts that several tens of muon induced neutrino events/year should be detectable with the IceCube neutrino telescope in coincidence with GRBs. Detection of the high energy neutrinos would not only test the shock acceleration mechanism, but would also imply GRBs are sources of Ultra High Energy Cosmic Rays (UHECR). A GRB at $100 \mathrm{Mpc}$ (for which the wide-field, high sensitivity EXIST trigger is needed) producing $100 \mathrm{TeV}$ neutrinos also allows a test of neutrino mass mixing five orders of magnitude more sensitive than solar neutrinos.

\section{Short GRBs and SGRs}

Short $(<2 \mathrm{sec})$ GRBs are still unidentified with afterglows or hosts and are thus without luminosities or redshifts. They are consistent with arising in NS-NS (or perhaps NS-BH) mergers [5]. EXIST should detect $\sim 10^{3}$ over a 3 year mission with fluxes above the BATSE threshold and locate these to $\sim 10$ " positional accuracy, sufficient to determine whether they are also (like long GRBs) associated with star formation regions in galaxies. If they arise from NS-NS mergers, or from accretion induced collapse of WDs [14], they may preferentially arise in globular cluster systems. Studies of the light curve variability enabled by the high statistics of EXIST can constrain short GRB jets (testing AIC), as it has apparently for long GRBs [15].

The soft gamma-ray repeaters (SGRs) are detected in the Galaxy (3) and LMC (1) and are associated with NSs, probably magnetars [16]. The Giant Flares detected from two of these reach highly superEddington luminosities which EXIST could detect with its all-sky monitoring and imaging out to $\sim 3-10 \mathrm{Mpc}$. This would survey SGRs and the incidence and activity of magnetars throughout the Local Group.

\section{CONCLUSIONS}

A Next Generation GRB mission to conduct the highest sensitivity direct study of GRBs would culminate the steady advance in sensitivity, resolution (spatial and spectral) and broad sky coverage, from BATSE through HETE-2 and Swift. The proposed EXIST mission would achieve this. It would provide the high sensitivity-resolution spectra and images for high energy GRBs from GLAST and afterglows from Constellation-X, NGST, LSST, and very large groundbased telescopes. As recommended by the Decadal Survey, EXIST could be launched by 2010 to open a new frontier in the study of the most extreme cosmic phenomena and the first stellar objects.

\section{REFERENCES}

1. Grindlay, J.E. et al., "EXIST: A High Sensitivity Hard X-ray Imaging Sky Survey Mission for ISS" in Proc. $5^{\text {th }}$ Compton Symposium, edited by M. McConnell and J. Ryan, AIP Conf. Proc., 510, 784 (2000).

2. Grindlay, J.E. et al., "EXIST: The Ultimate Spatial/Temporal Hard X-ray Survey", in Gamma 2001, eds. S. Ritz, N. Gehrels, and C. Shrader, AIP Conf. Proc., 587, 899 (2001)

3. Hartmann, D., MacFadyen, A. and Woosley, S., "The Most Distant Gamma-ray Bursts", in Proc. $5^{\text {th }}$ Huntsville Symposium, eds. R. Kippen, R. Mallozzi and G. Fishman,, AIP Conf. Proc., 526, 653 (2000).

4. MacFadyen, A. and Woosley, S., Ap. J., 524, 262 (1999).

5. Narayan, R., Piran, T. and Kumar, P., Ap. J., 557, 949 (2001).

6. Lamb, D. and Reichert, D.E., Ap. J., 536, 1 (2000).

7. Norris, J., Marani, G. and Bonnell, J., Ap. J., 534, 248 (2000).

8. Salmonson, J. and Galama, T., astro-ph/0112298 (2001).

9. Vanden Berk, D. et. al.., astro-ph/0111054 (2001).

10. Huang, Y., Dai, Z. and Lu, T., astro-ph/0112469 (2001).

11. Kippen. R. et. al., these proceedings (2002).

12. Schneider, R, Guetta, D. and Ferrara, A., astro$\mathrm{ph} / 0201342$ (2002).

13. Waxman, E. and Bahcall, J., Ap.J., 541, 707.

14. Usov, V., Nature, 357, 472 (1992).

15. Kobayashi, S., Ryde, F. and MacFadyen, A., astro$\mathrm{ph} / 0110080$ (2001).

16. Hurley, K. "Soft Gamma Repeaters in Review", in Proc. $5^{\text {th }}$ Huntsville Symposium, eds. R. Kippen, R. Mallozzi and G. Fishman,, AIP Conf. Proc., 526, 763 (2000). 